\title{
THE PROGENITOR MASS OF THE MAGNETAR SGR1900+14
}

\author{
Ben Davies $^{1,2}$, Don F. Figer ${ }^{2}$, Rolf-Peter Kudritzki ${ }^{3}$, Christine Trombley ${ }^{2}$, Chryssa Kouveliotou ${ }^{4}$, \\ AND STEFANIE WACHTER ${ }^{5}$ \\ ${ }^{1}$ School of Physics \& Astronomy, University of Leeds, Woodhouse Lane, Leeds LS2 9JT, UK \\ ${ }^{2}$ Chester F. Carlson Center for Imaging Science, Rochester Institute of Technology, 54 Lomb Memorial Drive, Rochester, NY 14623, USA \\ ${ }^{3}$ Institute for Astronomy, University of Hawaii, 2680 Woodlawn Drive, Honolulu, HI 96822, USA \\ ${ }^{4}$ Space Science Office, VP62, NASA/Marshall Space Flight Center, Huntsville, AL 35812, USA \\ ${ }^{5}$ Spitzer Science Center, 1200 E. California Blvd., California Institute of Technology, Pasadena, CA 91125, USA \\ Received 2009 September 3; accepted 2009 October 28; published 2009 November 25
}

\begin{abstract}
Magnetars are young neutron stars with extreme magnetic fields $\left(B \gtrsim 10^{14}-10^{15} \mathrm{G}\right)$. How these fields relate to the properties of their progenitor stars is not yet clearly established. However, from the few objects associated with young clusters it has been possible to estimate the initial masses of the progenitors, with results indicating that a very massive progenitor star $\left(M_{\text {prog }}>40 M_{\odot}\right)$ is required to produce a magnetar. Here, we present adaptive-optics assisted Keck/ NIRC2 imaging and Keck/NIRSPEC spectroscopy of the cluster associated with the magnetar SGR 1900+14, and report that the initial progenitor star mass of the magnetar was a factor of 2 lower than this limit, $M_{\text {prog }}=17 \pm 2 M_{\odot}$. Our result presents a strong challenge to the concept that magnetars can only result from very massive progenitors. Instead, we favor a mechanism which is dependent on more than just initial stellar mass for the production of these extreme magnetic fields, such as the "fossil-field" model or a process involving close binary evolution.
\end{abstract}

Key words: open clusters and associations: individual (Cl 1900+14) - stars: evolution - stars: individual (SGR1900+14) - stars: neutron

Online-only material: color figures

\section{INTRODUCTION}

Magnetars are currently recognized as a distinct group of neutron stars comprising of several classes of object, such as soft gamma repeaters (SGRs), anomalous X-ray pulsars (AXPs), and some compact central objects. These objects are characterized by relatively long spin periods and large spin-down torques, which imply magnetic fields on the order of $B \gtrsim 10^{14}-10^{15} \mathrm{G}$, making them the most highly magnetized objects known in the Universe (Duncan \& Thompson 1992; Thompson \& Duncan 1995; Kouveliotou et al. 1998; Mereghetti 2008). They are also known to enter active periods during which they emit very intense $\left(10^{37} \mathrm{erg} \mathrm{s}^{-1} \lesssim L \lesssim 10^{41} \mathrm{erg} \mathrm{s}^{-1}\right)$, short $(\sim 0.1 \mathrm{~s})$ hard $\mathrm{X}$-ray/gamma-ray bursts, as well as extremely energetic giant flares of $\gtrsim 10^{44}-10^{46}$ ergs lasting several minutes.

It is still unclear how magnetars are formed. The current theoretical framework for magnetar production requires that the core of a massive star has a very fast rotation speed in the first few seconds after it goes supernova (SN). If the rotation period is shorter than the convective timescale within the neutron starabout $1 \mathrm{~ms}$ - a highly efficient dynamo operates which boosts the magnetic field to $\sim 1000$ times that of a "regular" neutron star, and very rapidly slows the rotation period down to of order $1 \mathrm{~s}$ in a matter of years (the so-called "dynamo" mechanism; Duncan \& Thompson 1992; Thompson et al. 2004). However, recent stellar evolution calculations show that the cores of massive stars are substantially spun down as they enter the Red Supergiant (RSG) phase through magnetic braking between the stellar core and convective envelope (Heger et al. 2005). Thus, the problem exists of how the core of a massive star can retain sufficient angular momentum through to the $\mathrm{SN}$ stage such that the post-SN core is able to jump-start the dynamo mechanism. It has been suggested that those stars with $M_{\text {init }} \gtrsim 40 M_{\odot}$ are able to lose a substantial fraction of their hydrogen-rich envelope while still on the main sequence, allowing them to skip the RSG phase, and therefore avoid the severe spin-down of the core as the outer envelope expands and becomes convective (Gaensler et al. 2005b).

Where magnetars have been associated with star clusters, it has been possible to estimate the initial mass of the progenitor empirically. Evidence suggests that the magnetar phase is short and that the SN that produced it must have occurred recently $\left(\lesssim 10^{4} \mathrm{yr}\right.$ ago; Kouveliotou et al. 1994), such that the age of the cluster is much greater than the lifetime of the magnetar. Consequently, by measuring the age of the star cluster, we can determine the age of the progenitor star when it went SN. Then, as a star's lifetime is a strong function of its initial mass, we can estimate the initial mass of the magnetar's progenitor. In the cases of the magnetars SGR $1806-20$ and CXOU J164710.2-455216, associated with the clusters $\mathrm{Cl} 1806-20$ and Westerlund $1(\mathrm{Wd} 1)$, respectively, it appears that the magnetar progenitors had initial masses $\gtrsim 40 M_{\odot}$ (Figer et al. 2005; Bibby et al. 2008; Muno et al. 2006). These results are therefore consistent with the hypothesis that magnetars descend from the most massive stars. Further supporting evidence for this hypothesis comes from a study of the expanding $\mathrm{H}$ I shell around the magnetar 1E 1048.1-5937. When the shell was interpreted as a stellar wind bubble blown by the progenitor, a progenitor mass of 30-40 $M_{\odot}$ was inferred (Gaensler et al. 2005a).

There is a fourth magnetar, SGR $1900+14$, which can be used to test this hypothesis. It too is thought to belong to a cluster, which was first recognized for its two bright RSG members (Vrba et al. 1996, 2000). However, up until now this cluster has been poorly studied. The only current distance estimate is a spectrophotometric distance that assumes an intrinsic brightness for the RSGs, and so cannot be used to derive accurate luminosities for the RSGs themselves. Consequently, any estimate for the magnetar's progenitor mass based on the RSG luminosities is unreliable. The best evidence for the association of the magnetar and star cluster comes from the 
detection of an infrared ring around the source, analysis of which placed the magnetar at the same distance from Earth as the cluster's spectrophotometric distance (Wachter et al. 2008).

In this paper, we present a spectroscopic and photometric analysis of the stellar content of the cluster $\mathrm{Cl} 1900+14$. We determine a kinematic distance to the cluster and derive the cluster's age. Ultimately, we are able to establish an estimate for the initial mass of the progenitor of SGR 1900+14. We begin in Section 2 with a description of the observations and data reduction procedure. In Section 3, we present the results of our analysis; and in Section 4, we discuss the implication for the evolution of massive stars.

\section{OBSERVATIONS AND DATA REDUCTION}

\subsection{Spectroscopy}

Spectroscopic observations were made using NIRSPEC, the infrared spectrograph mounted on Keck-II, during the night of 2008 June 23. High-resolution observations were made of the stars A and B (as identified by Vrba et al. 2000) with the spectrograph in cross-dispersed mode, the cross-disperser angle set to 35.53 and the echelle angle to 62.53 . We used the NIRSPEC-7 filter and the 0 '. $576 \times 24^{\prime \prime}$ slit, which gave a spectral resolution of $R \approx 20,000$ in select regions of the $K$ band.

Low-resolution observations were also made of several other stars within the field of the cluster. Here, the echelle was replaced with a mirror, which when combined with the cross-disperser and the $42^{\prime \prime} \times 0.57$ slit gave a spectral resolution of $R=1000$ in the wavelength range $1.9-2.4 \mu \mathrm{m}$.

Repeated observations were made of each target, with the star nodded along the slit by $\gtrsim 10^{\prime \prime}$. In addition to the target stars, we also observed the telluric standard stars HD179282 and HD173003 to characterize the atmospheric absorption features in the $K$ band. Continuum-lamp exposures were made for flat-field purposes, arc lamps were observed for wavelength calibration, while in high-resolution mode we also observed the continuum lamp through the etalon filter to sample the gaps in between the arc lines.

Our data reduction procedure included the subtraction of nodpairs to remove diffuse sky emission, and division by a normalized flat-field frame to correct for variations in pixel sensitivity on the detector. Before extraction, the spectral traces were resampled onto a grid linear in both the spatial and dispersion directions using the arc and etalon frames. This rectification process provides absolute wavelength calibration accurate to $\pm 4 \mathrm{~km} \mathrm{~s}^{-1}$ for the high-resolution spectra-a detailed description of this part of the data reduction is given in Figer et al. (2003). Atmospheric absorption features were removed by dividing through by a telluric standard star, which first had its intrinsic spectral features removed using a synthesized spectrum appropriate for its spectral type.

\subsection{Imaging}

The field centered on the cluster was imaged using the Near Infrared Camera 2 (NIRC2) on Keck-II, in combination with Laser Guide Star Adaptive Optics (LGSAO), on 2008 August 23. Five overlapping $20 \mathrm{~s}$ exposures were taken in the $H$ and $K$ bands, using the narrow camera, in a X-shaped dither pattern. Raw images were cleaned by subtraction of dark and flat fielding, then median combined.

Aperture photometry of point sources was done using IDL scripts, including those in the IDL-adapted version of

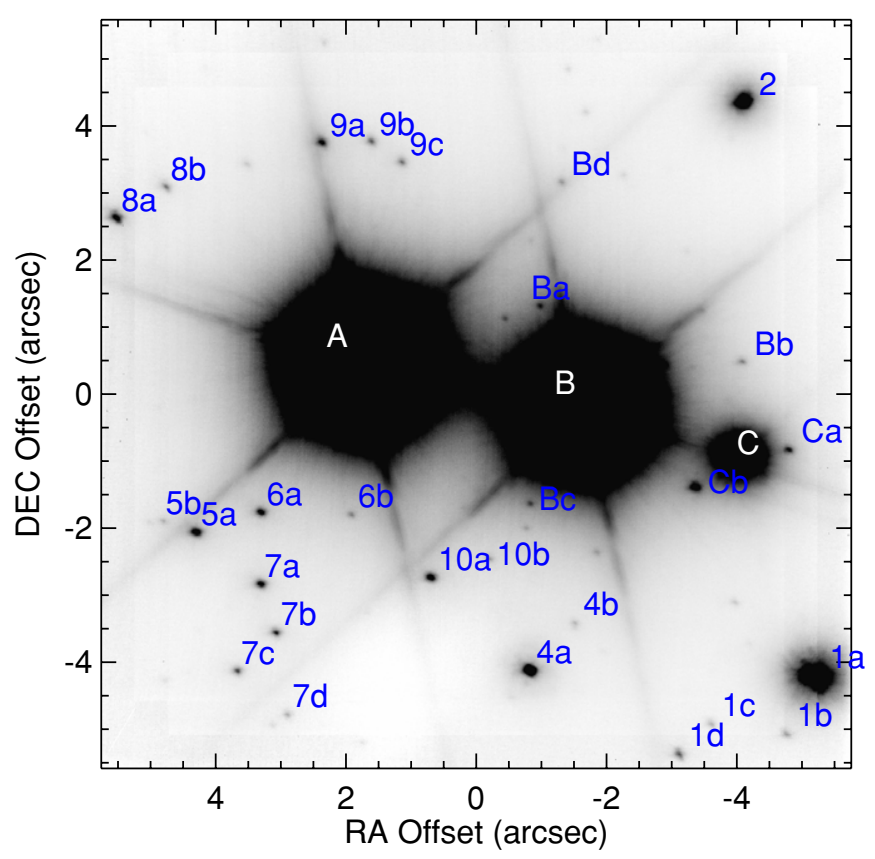

Figure 1. NIRC2 $K$-band image of the center of the field, showing the fainter stars close to the two RSGs. We use the stellar identifications of Vrba et al. (2000); where we resolve one of Vrba et al.'s objects into multiple components, and sub-label them alphabetically.

(A color version of this figure is available in the online journal.)

DAOPHOT. Point sources that were too close to the two saturated bright stars in the center were rejected. Magnitudes were calibrated from a control frame taken the same night, and by using Two Micron All Sky Survey (2MASS) photometry of bright, isolated, non-saturated stars. For astrometric calibration, we used archival Hubble Space Telescope (HST) observations of a field containing the cluster, and used stars 9a, 7a, and 4 (see Figure 1) to compute the geometric transformation.

In addition to the Keck/NIRC2 photometry, we also obtained photometry of stars over a wider field from the UKIDSS survey of the Galactic plane (Lucas et al. 2008; Lawrence et al. 2007; Casali et al. 2007; Hewett et al. 2006; Hambly et al. 2008). In Figure 2, we show this wide-field image, and indicate the stars for which we obtained spectroscopy.

\section{RESULTS AND ANALYSIS}

The high-resolution $K$-band spectra of the two stars A and B in the region of the $\mathrm{CO}$ band head are presented in Figure 3, with both objects displaying the deep absorption signature of luminous late-type stars. The star "C" was shown by V96 to be a foreground object. To derive physical parameters for the two stars, we follow the method of Davies et al. (2007). To determine a spectral type for each star, we measured the equivalent width of the $\mathrm{CO}$ band head and compared to similar measurements of template stars. We found absorption strengths that were in excess of that seen in giant stars, and were more typical of M1-2 supergiants. Below we describe our analysis procedure in more detail, the results of which are presented in Table 1.

\subsection{Extinction}

We measured line-of-sight extinctions from the $J-K$ and $H-K$ color-excesses of the two stars, using the photometry of Vrba et al. (1996), intrinsic colors from Levesque et al. (2005) appropriate for the stars' spectral types, and the extinction law 
Table 1

Physical Properties of the Red Supergiants in Cl 1900+14

\begin{tabular}{lcccccc}
\hline \hline Star & $\begin{array}{c}v_{\text {LSR }} \\
\left(\mathrm{km} \mathrm{s}^{-1}\right)\end{array}$ & $\begin{array}{c}T_{\text {eff }} \\
(\mathrm{K})\end{array}$ & $\begin{array}{c}\text { Spec Type } \\
( \pm 2 \text { subtypes })\end{array}$ & $A_{\mathrm{K}}$ & $M_{\mathrm{K}}$ & $\log \left(L / L_{\odot}\right)$ \\
\hline A & $-14 \pm 4$ & $3660 \pm 130$ & $\mathrm{M} 2$ & $1.43 \pm 0.06$ & $-10.64_{-0.19}^{+0.20}$ & $5.05_{-0.08}^{+0.08}$ \\
B & $-17 \pm 4$ & $3750 \pm 120$ & M1 & $1.45 \pm 0.03$ & $-10.23_{-0.17}^{+0.18}$ & $4.91_{-0.07}^{+0.07}$ \\
\hline
\end{tabular}

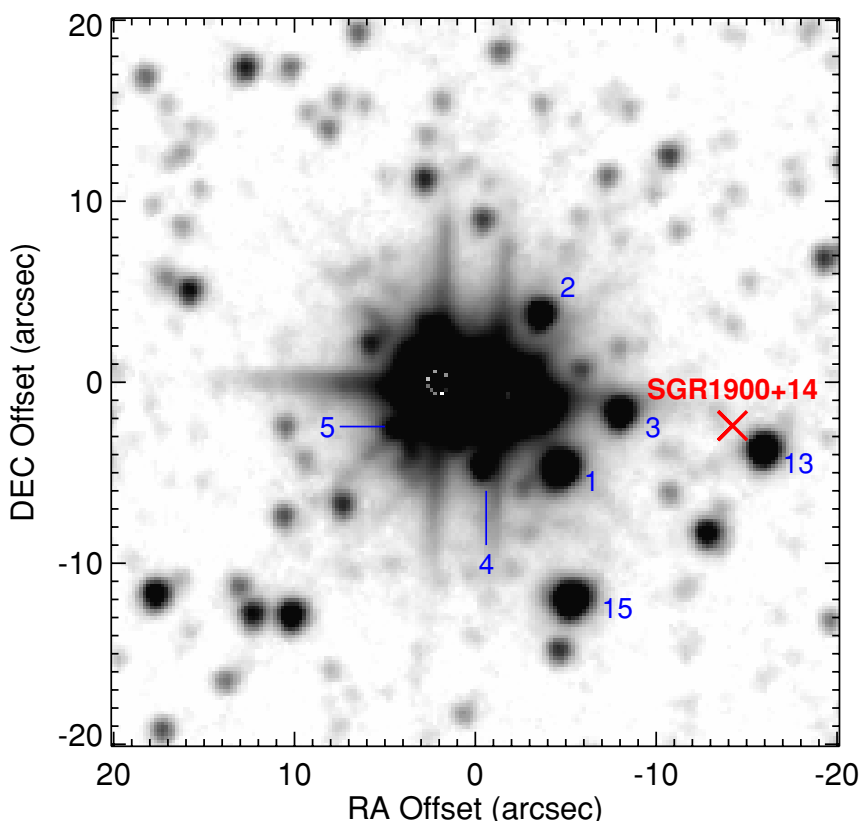

Figure 2. Wide-field UKIDSS $H$-band image of $\mathrm{Cl} 1900+14$, indicating the stars of which low-resolution spectra were obtained. The position of the magnetar SGR1900+14 is indicated.

(A color version of this figure is available in the online journal.)

of Rieke \& Lebofsky (1985). We found comparable extinctions for both stars from each IR color. The $H-K$ extinction for the two RSGs, $A_{K}=1.44 \pm 0.06$, implies a visual extinction of $A_{V}=12.9 \pm 0.5$ if we assume $A_{K} / A_{V}=0.112$ (Rieke $\&$ Lebofsky 1985). This is in excellent agreement with the extinction derived by Kaplan et al. (2002), $A_{V}=12.8 \pm 0.8$, determined from converting $\mathrm{X}$-ray absorption to an equivalent hydrogen column density. However, it is very different from the extinction derived by Vrba et al. (1996), who found $A_{V} \simeq 19.2$. The reason that such a large extinction was inferred by these authors is not clear (see also Wachter et al. 2008), however it may be that the extinction law they used was optimized for optical wavelengths while they used near-IR color indices to determine the reddening.

\subsection{Kinematic Distance}

We measured radial velocities for the two stars by crosscorrelating the spectra with that of Arcturus presented in Wallace \& Hinkle (1996), which had been degraded to the same spectral resolution as our observations and shifted to the rest frame. As with extinction, the line-of-sight velocities we measure for the two RSGs are comparable to within the errors. This is strong evidence that the objects are part of a physical association. The mean velocity we measure, ${ }^{6}-15.5 \pm 4 \mathrm{~km} \mathrm{~s}^{-1}$, indicates a

\footnotetext{
6 The uncertainty on the velocity is dominated by the absolute precision of
} our measurements.

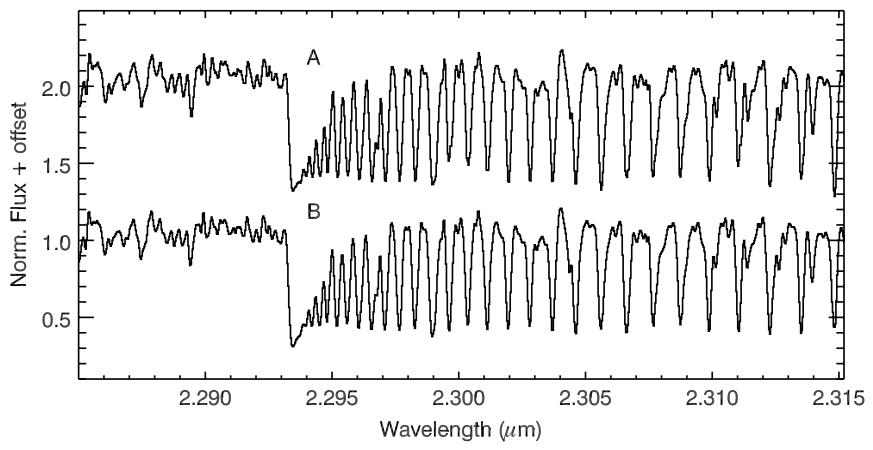

Figure 3. High-resolution spectra of the two RSGs in the region of the CO band head absorption feature.

kinematic distance of $12.5 \pm 0.3 \mathrm{kpc}$ using the Galactic rotation curve of Brand \& Blitz (1993). As we measure a negative velocity, there is no nearside solution to the rotation curve along this line of sight. Though random velocities which are peculiar to the Galactic rotation curve by up to $20 \mathrm{~km} \mathrm{~s}^{-1}$ may not be uncommon, this would require the cluster to be within $1 \mathrm{kpc}$, which given the extinction to the object is highly unlikely. If we take such a random motion as the dominant source of uncertainty in the kinematic distance, we find $12.5 \pm 1.7 \mathrm{kpc}$. This distance is in good agreement with the spectrophotometric estimate by Vrba et al. (1996).

\subsection{Cluster Age}

The distance, average extinction, and the bolometric corrections of Levesque et al. (2005) were used to calculate intrinsic luminosities of the RSGs in the cluster. As a cluster ages, the stars currently experiencing the RSG phase will have descended from stars with lower initial masses, and so will have lower luminosities. Therefore, from the luminosity range of the RSGs in a cluster it is possible to estimate the cluster's age.

Figure 4 shows the minimum and maximum luminosities of RSGs in a coeval cluster, as a function of cluster age, calculated using the Geneva rotating models at Solar metallicity (Meynet \& Maeder 2000). The initial masses of the stars in the RSG phase are labeled. The red arrows indicate the range of RSG luminosities we observe in $\mathrm{Cl} 1900+14, \pm 1 \sigma$. We can say that the cluster cannot be younger than $\approx 13 \mathrm{Myr}$, as the least luminous RSG in such a cluster would be brighter than the faintest RSG in $\mathrm{Cl} 1900+14$. Similarly, we can place an upper limit to $\mathrm{Cl} 1900+14$ 's age of $\approx 15 \mathrm{Myr}$, as the RSGs in a cluster older than this would be fainter than the brightest $\mathrm{RSG}$ in $\mathrm{Cl} 1900+14$. Therefore, from the RSG luminosity range we observe in the cluster, we can constrain the age to $14 \pm$ 1 Myr. If a greater number of RSGs were to be present in this cluster, we would expect them to occupy the luminosity range indicated by the shaded region between 13-15 Myr $\left(4.75<\log \left(L / L_{\odot}\right)<5.25\right)$. The effect of using different evolutionary models is discussed in Section 4. 


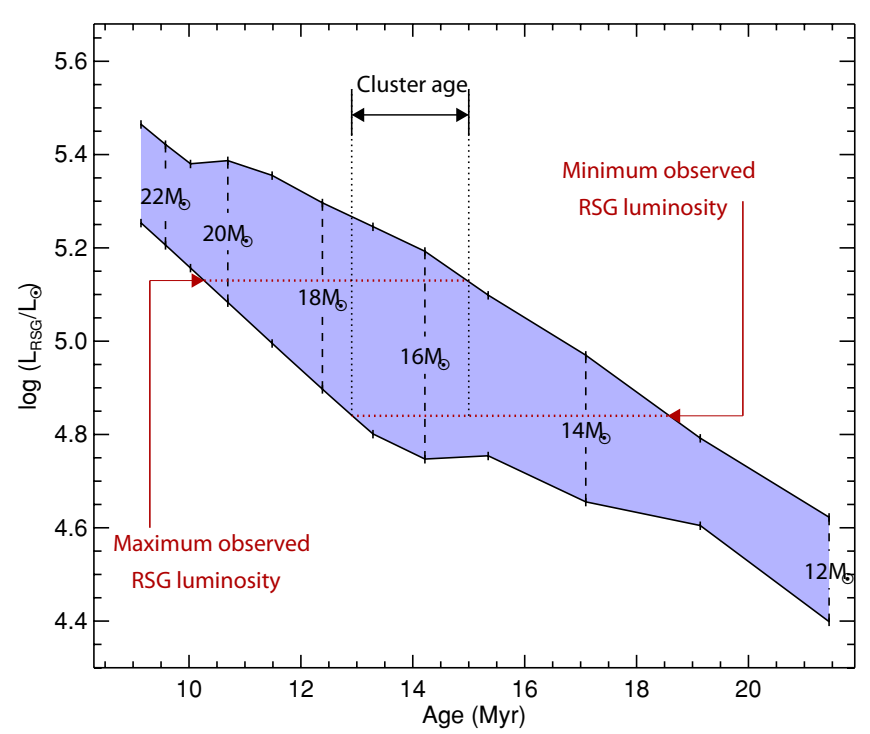

Figure 4. Minimum and maximum luminosities of Red Supergiants (RSGs) in a coeval cluster, as a function of cluster age, calculated using the Geneva rotating models at Solar metallicity Meynet \& Maeder (2000). The initial masses of the stars in the RSG phase are labeled. The red arrows indicate the range of RSG luminosities we observe in $\mathrm{Cl} 1900+14, \pm 1 \sigma$.

(A color version of this figure is available in the online journal.)

Table 2

Stars Observed at Low Spectral Resolution, with Photometry and Rough Spectral Types

\begin{tabular}{lcccc}
\hline \hline Star & $J$ & $H$ & $K$ & Spec Type \\
\hline V-C & 12.47 & 11.30 & 10.45 & K/M \\
V-1a & 13.07 & 11.91 & 11.17 & K/M \\
V-2 & 14.22 & 13.02 & 12.26 & K/M \\
V-3 & 14.51 & 12.86 & 12.05 & O/B \\
V-4a & 14.63 & 13.25 & 12.38 & K/M \\
V-5a & $\ldots$ & 15.19 & 14.41 & O/B \\
V-13 & 14.25 & 12.68 & 11.98 & O/B \\
V-15 & 13.67 & 12.12 & 11.36 & O/B \\
\hline
\end{tabular}

Notes. Stellar identifications are taken from Vrba et al. (2000). Photometry is taken from UKIDSS, with the exception of V-C (from Vrba et al. 1996) and V-5a (from NIRC2 photometry presented in this paper).

\subsection{Cluster Coevality}

We can assess the coevality of the cluster by studying its stellar population. From our low-resolution spectroscopy of stars in the cluster field, we separate the massive stars belonging to the cluster from foreground objects using the spectral signatures of massive stars. That is, massive stars are hot, their spectra displaying features attributable to transitions of hydrogen and helium; while foreground low-mass stars are cool and have features attributable to molecular $\mathrm{CO}$.

In Table 2, we list the stars in close proximity to the RSGs $\mathrm{A}$ and $\mathrm{B}$ that were observed at low spectral resolution. We also tabulate the photometry of these stars-where none was available in the literature or from our NIRC2 data-and obtained photometry from the UKIDSS Galactic Plane Survey Lucas et al. (2008). We assigned coarse spectral types to the objects based on the presence of $\mathrm{CO}$ band head absorption - those with $\mathrm{CO}$ were classified as late-type $(\mathrm{K} / \mathrm{M})$; and those without as early-type $(\mathrm{O} / \mathrm{B})$.

In Figure 5, we plot the spectra of the "early-type" stars. All stars exhibit clear spectral features of $\mathrm{Br}-\gamma+\mathrm{He}$ I $(2.161,2.164)$,

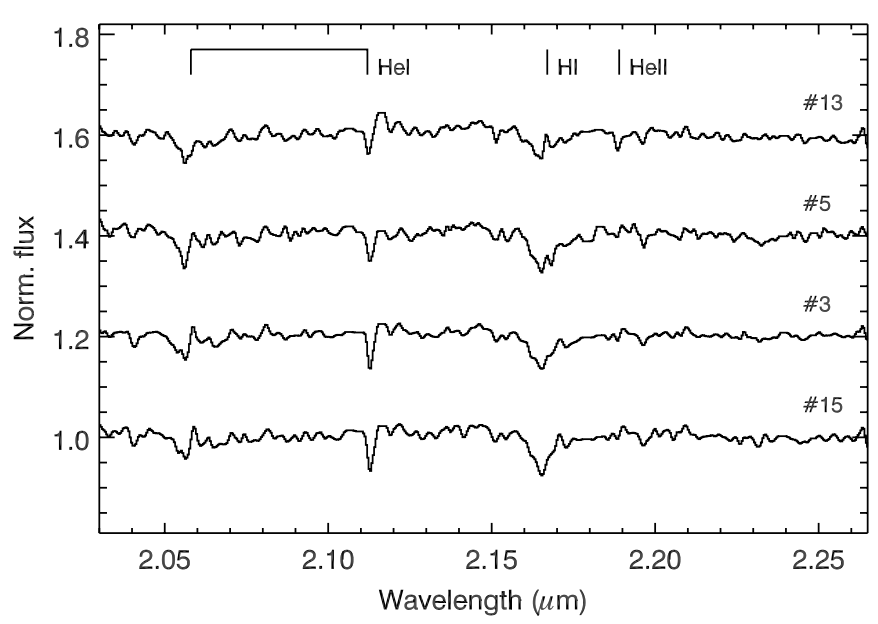

Figure 5. Low-resolution spectra of early-type stars in the cluster.

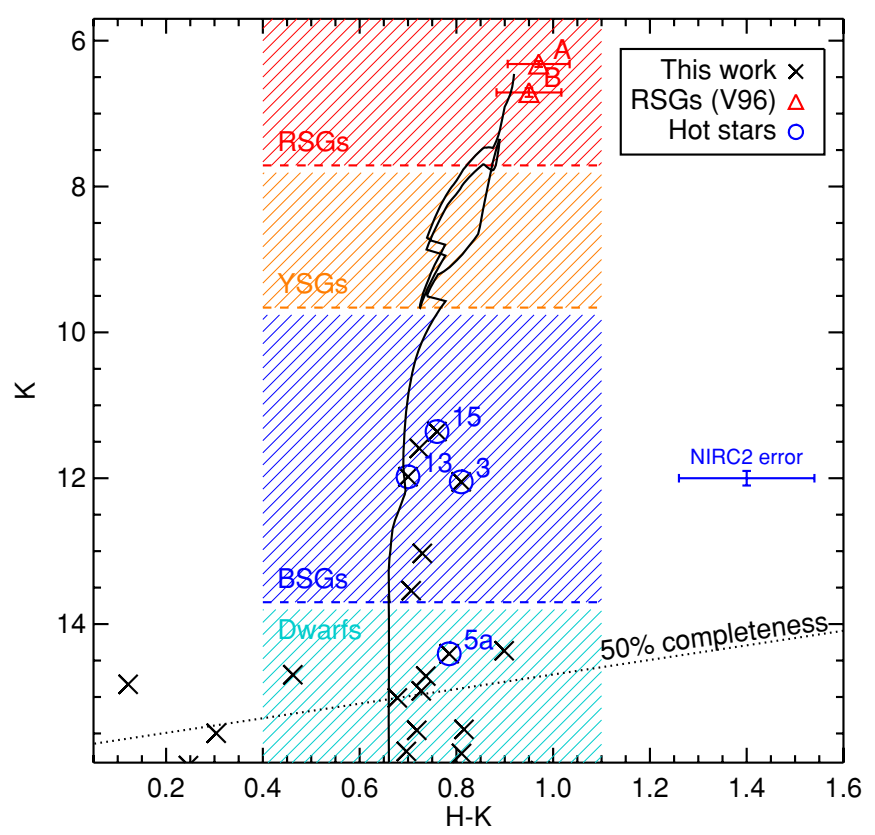

Figure 6. Color-magnitude diagram for stars in the NIRC2 field of view, including the photometry from V96. Overplotted is an $15 \mathrm{Myr}$, solar-metallicity isochrone from Schaller et al. (1992), using the distance and extinction derived in this paper. Indicated on the plot are the magnitude ranges for RSGs, YSGs, BSGs, and dwarfs in a cluster of that age, distance, and extinction.

(A color version of this figure is available in the online journal.)

He $\mathrm{I}-2.112 \mu \mathrm{m}$, and He $\mathrm{I}-2.058 \mu \mathrm{m}$. There is a suggestion of He II-2.189 $\mu \mathrm{m}$ absorption in the spectrum of No. 13, though the strength of this feature is comparable to the noise. To spectraltype the stars, we compare to the spectral atlases of Hanson et al. (1996) and Hanson et al. (2005). The absence of any strong He II-2.189 $\mu \mathrm{m}$, as well as a lack of C IV and N III, suggests that the stars cannot be any earlier than $\sim 07$. Also, the comparable strengths of the $\mathrm{Br}-\gamma+\mathrm{He} \mathrm{I}(2.161,2.164) \mu \mathrm{m}$ suggests that the stars are no later than $\sim \mathrm{B} 3$. At this spectral resolution, it is not possible to distinguish between dwarfs and supergiants using the width of $\mathrm{Br}-\gamma$ as it blends with the adjacent $\mathrm{He}$ I feature.

In Figure 6, we show the color-magnitude diagram (CMD) for the NIRC2 field. We also show the photometry of the three bright stars from V96. A $15 \mathrm{Myr}$ isochrone from the non-rotating 


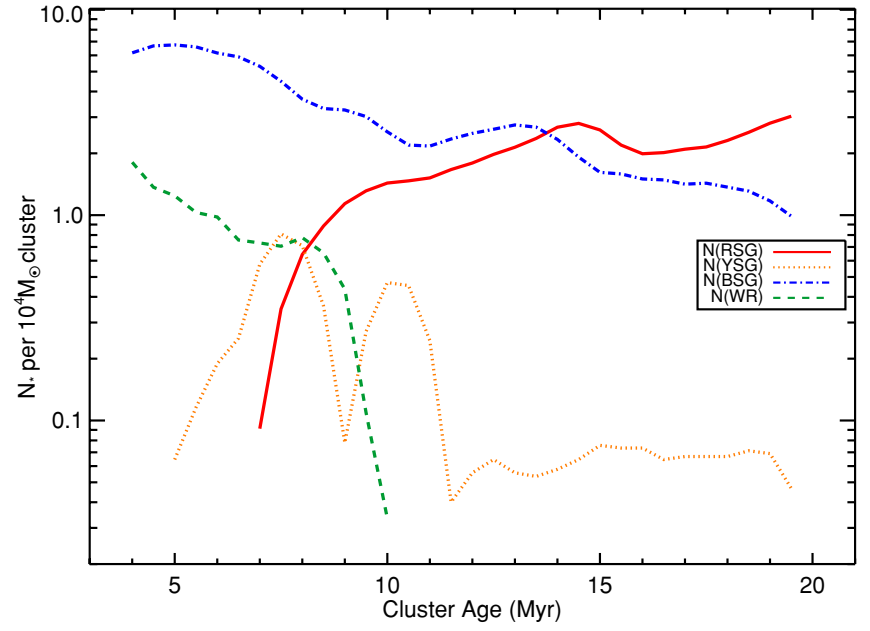

Figure 7. Relative numbers of BSGs, RSGs, YSGs, and WRs in a coeval cluster of mass $10^{4} M_{\odot}$ as a function of cluster age.

(A color version of this figure is available in the online journal.)

Geneva tracks (Schaller et al. 1992) ${ }^{7}$ is overplotted, using the extinction and distance derived in Section 3.1.

In the panel, we have indicated the apparent $K$-band magnitude ranges for RSGs, Blue Supergiants (BSGs), Yellow Supergiants (YSGs), and dwarf stars for a cluster with this age, distance, and extinction, using the Schaller et al. (1992) models. They are defined as follows:

1. RSGs: $T_{\text {eff }}<4000 \mathrm{~K}, L_{\star}>10^{4} L_{\odot}$;

2. YSGs: $10,000 \mathrm{~K}>T_{\text {eff }}>4000 \mathrm{~K}, L_{\star}>10^{4} L_{\odot}$;

3. BSGs: $T_{\text {eff }}>10,000 \mathrm{~K}, \log g<3.5, L_{\star}>10^{4} L_{\odot}$;

4. Dwarfs: ${ }^{8} \log g>3.5, L_{\star}<10^{4} L_{\odot}$.

The plot shows that, though there are two RSGs in the cluster, there are no stars with brightnesses consistent with being YSGs. There are some objects with luminosities one would expect to see of BSGs-however, several of these stars have been spectroscopically identified as cool stars, and are therefore likely foreground objects. Stars, which show signatures of early spectral types, are indicated in Figure 6 as circles. Overall we find three stars with $K$-band magnitudes consistent with BSGs and one star consistent with a main-sequence object.

In comparing the relative fractions of the different evolutionary stages with those predicted by the evolutionary track, we find good agreement. Using models with ages 13-17 Myr from Schaller et al. (1992) and an initial mass function (IMF) defined by Kroupa (2001), RSGs are predicted to outnumber YSGs by a factor of $\gtrsim 10$. The ratio of the number of RSGs to BSGs is subject to large variations depending on the age of the cluster, and can be between 0.1 and 10 in the age range we determine for the cluster. This is also illustrated in Figure 7, where we use the updated evolutionary models of Meynet \& Maeder (2000), which include stellar rotation to determine the relative fractions of evolved massive stars in a coeval cluster as a function of age.

Thus, we can say that we are unlikely to find any YSGs in a cluster that contained only two RSGs, while the number of BSGs should be within an order of magnitude of the number of RSGs. In addition, we find that there should be approximately 10 times as many main-sequence stars brighter than our $50 \%$

\footnotetext{
7 We use the rotating models of Meynet \& Maeder (2000) for most of our analysis, which at the moment are not available with instrumental colors.

8 We use $\log g$ as a classification criterion in order to distinguish between main-sequence and post main-sequence objects.
}

completeness limit at $K=15$. While we have only identified one such star here, there are many more B-dwarf candidates in the NIRC2 field of view which occupy the relevant region of the CMD and which await spectroscopic classification.

From this analysis, we conclude from the luminosities of the RSGs, the absence of WRs, and the relative numbers of $N(\mathrm{RSG}) / N(\mathrm{BSG})$, that $\mathrm{Cl} 1900+14$ is fully consistent with being a $14 \pm 1 \mathrm{Myr}$, coeval cluster. By extrapolating over the rest of a Kroupa IMF down to $0.01 M_{\odot}$, we estimate that the total cluster mass of this cluster is $\sim 10^{3} M_{\odot}$ to within an order of magnitude.

\section{THE INITIAL MASS OF THE MAGNETAR'S PROGENITOR}

To summarize the results of the previous section, in terms of the cluster's age we find that the RSG luminosities are uniquely fitted by the rotating stellar evolutionary models at Solar metallicity Meynet \& Maeder (2000) for an age of $14 \pm$ 1 Myr. Analysis of the fainter stars indicate that the cluster is consistent with being a coeval starburst to within the errors. No Wolf-Rayet stars are found, which would imply star formation within the last $\sim 8 \mathrm{Myr}$, while we find relative numbers of hot/ cool stars that are consistent with the model predictions for a coeval 14 Myr cluster. As the age of the cluster is much greater than the lifetime of the magnetar $\left(\lesssim 10^{4} \mathrm{yr}\right)$, we can now estimate the mass of the magnetar's progenitor by determining the mass of the most massive star that could still exist in a cluster of this age. Using the same stellar evolution models as above we find that the initial mass of the magnetar's progenitor was $M_{\text {prog }}=17 \pm 1 M_{\odot}($ see Figure 4$)$.

In Figure 8, we examine the robustness of this result by exploring the effects of using different evolutionary models to determine the cluster age. In the top-left panel of Figure 8, we show the result of using the updated Geneva models which do not include rotation (Meynet \& Maeder 2000). As discussed by Meynet $\&$ Maeder, one effect of including rotation in their evolutionary code is that the post main-sequence massive stars become more luminous. Hence, for a given stellar luminosity, the inferred age from rotating models is larger than for nonrotating models. We see from the top-left panel of Figure 8 that when the non-rotating models are used, the cluster age is reduced to 9-10 Myr. However, the implied initial masses of the RSGs are altered only slightly- $19 M_{\odot}$, as opposed to $16-$ $17 M_{\odot}$ from the rotating models.

In the remaining panels of Figure 8 we explore the use of earlier models of the Geneva group, which do not include rotation but which do include convective overshooting. We see that, while inferred cluster ages are systematically lower than those determined using rotating models, the RSG masses are consistently in the region of $17-19 M_{\odot}$. This is the case whether the metallicity is Solar, twice-Solar or Solar/2.5. Similarly, doubling the stellar mass-loss rates has little impact.

From this analysis we conclude that the version of evolutionary model used to calculate the initial mass of the magnetar's progenitor has very little impact on the value derived. From the contemporary models, which include rotation, we find $M_{\text {prog }}=17 \pm 1 M_{\odot}$; while from earlier variations of the Geneva evolutionary code we consistently find masses in the range of 17-19 $M_{\odot}$. Therefore, we find that any systematic uncertainty in $M_{\text {prog }}$ must be small, of order 1-2 $M_{\odot}$. To account for systematic uncertainties, we adopt an error of $\pm 2 M_{\odot}$ on the derived progenitor mass. 

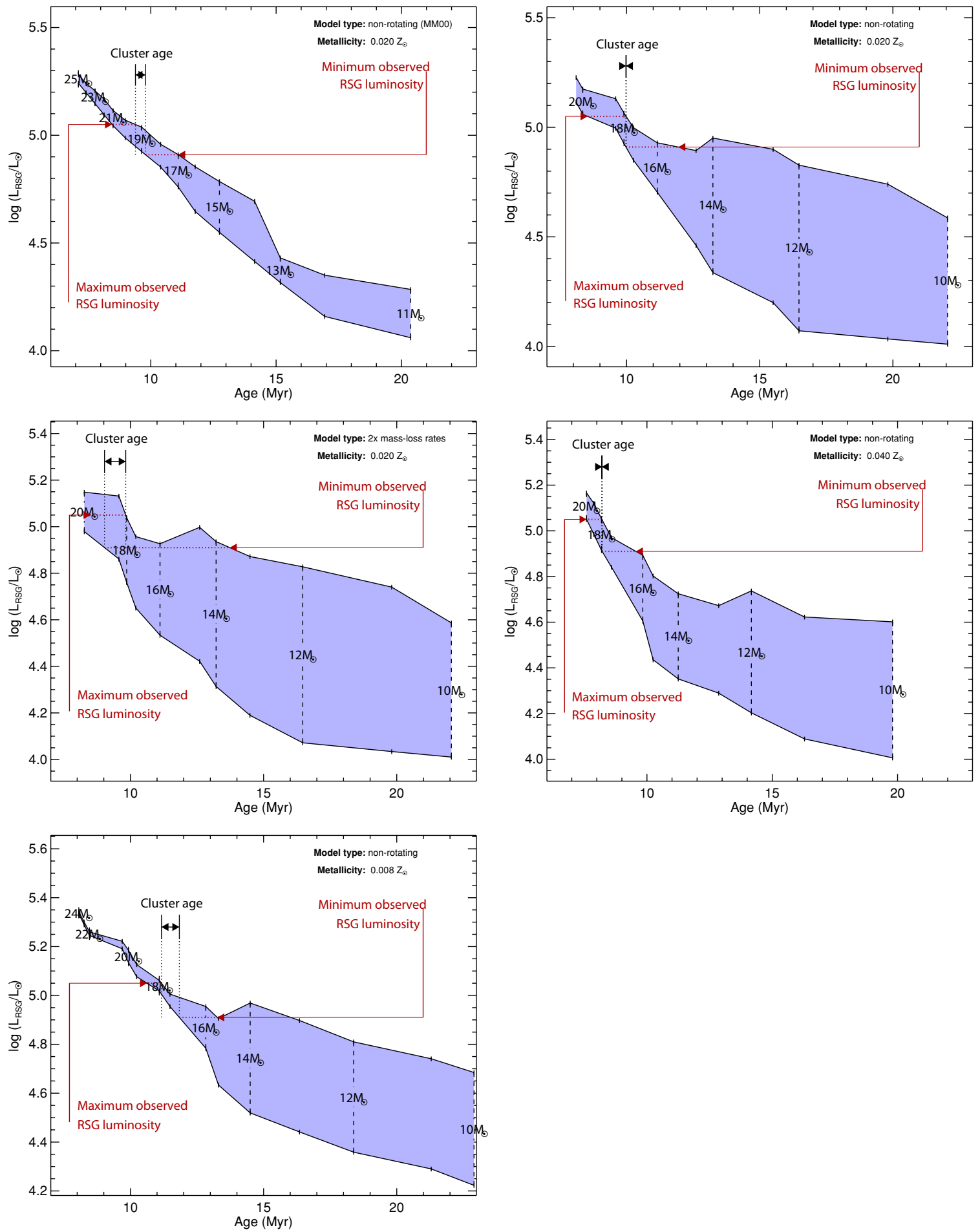

Figure 8. Similar figures to that of Figure 4. The figures illustrate the implied age of a cluster containing RSGs with the luminosity range we observe for Cl $1900+14$ when different evolutionary models are used. Top left: non-rotating models (Meynet \& Maeder 2000); top-right: obsolete non-rotating models (Schaller et al. 1992); middle-left: same as top-right but with doubled mass-loss rates (Meynet et al. 1994); middle-right: same as top-right but twice-Solar metallicity (Schaerer et al. 1993); and bottom-left: same as top-right but for LMC metallicity (Schaerer et al. 1993).

(A color version of this figure is available in the online journal.)

\subsection{The Possible Role of Binarity}

We now consider the role that binarity may play in magnetar production. To recap, one supporting piece of evidence cited by Gaensler et al. (2005a) for high progenitor masses of magnetars was from the numerical simulations of Heger et al. (2005). These authors found that the presence of internal magnetic fields may work to transport angular momentum away from the core, particularly during the transition to core He burning, as the star adjusts to the large differential rotation between the core and the inflated envelope of the RSG phase. A progenitor with initial mass similar to that we derive for SGR $1900+14$ would almost certainly pass through the RSG phase as a single star. However, if a star were to evolve in a close binary system, it has been 
Table 3

Post-supernova Objects for which Progenitor Masses can be Estimated from their Host Clusters

\begin{tabular}{lcccc}
\hline \hline \multicolumn{1}{c}{ Object [+ Cluster] } & $M_{\text {prog }} / M_{\odot}$ & Remnant & $B\left(\times 10^{14} \mathrm{G}\right)$ & Ref. \\
\hline SGR 1806-20 & $48_{-8}^{+20}$ & Magnetar & $2-8$ & 1,2 \\
CXO J164710.2-455216 [Wd 1] & $40 \pm 5$ & Magnetar & $<1.5$ & 3 \\
IGR J18135-1751 [Cl 1813-18] & $20-30$ & Pulsar Wind Nebula & 0.03 & $4,5,6$ \\
AX J1838-0655 [RSGC1] & $18 \pm 2$ & Pulsar Wind Nebula & 0.02 & 7,8 \\
SGR 1900+14 & $17 \pm 1$ & Magnetar & $2-8$ & This work, 9
\end{tabular}

References. (1) Bibby et al. 2008; (2) Kouveliotou et al. 1998; (3) Muno et al. 2006; (4) Helfand et al 2007; (5) Messineo et al. 2008; (6) Gotthelf \& Halpern 2009; (7) Davies et al. 2008; (8) Gotthelf \& Halpern 2008; (9) Kouveliotou et al. 1999.

shown that the effects of mass transfer onto a companion star significantly reduce the RSG lifetime (Eldridge et al. 2008). This enables the core to retain angular momentum through to SN, enough perhaps to greatly amplify the resulting neutron star's magnetic field through the dynamo mechanism in the first few seconds of its life. In addition, the presence of a secondary star may serve to add angular momentum to the primary. We note, however, that in this scenario the secondary star would remain after the primary had gone SN. As yet, there is no compelling evidence for an optical/near-IR counterpart to SGR1900+14 (a faint candidate with $K=19.21$ was suggested by Testa et al. 2008).

\subsubsection{Was the Progenitor the Product of a Merger?}

If the magnetar's progenitor star was part of a binary system that merged before the primary went SN, this could explain the lack of any obvious optical/IR counterpart. Indeed, such a process may seem appealing from the point of view of magnetar production; it would increase the terminal mass of the star, while it would also likely spin-up the core. If such a merger did occur it must have been at a time shortly before the $\mathrm{SN}$ explosion that created the magnetar. Otherwise, the lifetime of the resulting more massive star would be significantly shortened and would have gone SN many millions of years ago. Hence, the time since $\mathrm{SN}$ would be much greater than current estimates of magnetar lifetimes $\left(10^{4} \mathrm{yr}\right)$.

Such an explanation may seem contrived, however a similar scenario has been proposed for the progenitor of SN1987A, where two stars of masses $\sim 15 M_{\odot}$ and $\sim 5 M_{\odot}$ merged around $20,000 \mathrm{yr}$ prior to SN (see, e.g., Podsiadlowski et al. 1992). It is commonly thought that mergers of stars in binary systems are far more likely to occur in systems with large mass ratios. In order to merge, the primary must evolve off the main sequence such that the secondary is enveloped in the primary's inflated atmosphere, whereupon the two stars spiral downward toward the center of mass. Hence, if a merger did occur in the progenitor of SGR1900+14, it is unlikely that the pre-merger system consisted of two $17 M_{\odot}$ stars and was more likely a $17 M_{\odot}$ primary and a secondary of mass $\lesssim 10 M_{\odot}$.

We cannot discount the possibility that the progenitor of SGR1900+14 experienced a merger, nor can we rule out that such mergers are a necessary ingredient in the magnetar production mechanism. However, the evidence for such a merger is limited at best. While the magnetar is at the center of a largescale ring nebula (Wachter et al. 2008), and a similar ring around SN1987A is proposed to be the product of a merger prior to SN (Morris \& Podsiadlowski 2006), mergers are not the only mechanism to produce such rings. This alone is not compelling evidence for a merger event for the progenitor of SGR1900+14.

\subsection{Comparison with Other Neutron Stars with Known Progenitor Masses}

How does our mass measurement of $17 M_{\odot}$ compare to other post-SN objects with progenitor mass estimates? In Table 3, we list all known young clusters associated with neutron stars. As well as the three clusters containing magnetars, we also list the two recent discoveries of clusters associated with Pulsar Wind Nebulae (PWNe) - Cl 1813 - 13 (Helfand et al. 2007; Messineo et al. 2008; Gotthelf \& Halpern 2009), and RSGC1 (Davies et al. 2008; Gotthelf \& Halpern 2008). Prior to our current result, it could be argued from these data that there is a connection between magnetic field strength $B$ and progenitor mass. However, the inclusion of SGR 1900+14-the object with the lowest progenitor mass of the sample, but whose magnetic field is as large as any other on the list-appears to end any notion of a relation between $B$ and $M_{\text {prog }}$. As such, our result provides a strong challenge to the hypothesis that magnetars descend from very massive stars-specifically, those stars massive enough to avoid the RSG phase and the associated core spin-down (Heger et al. 2005). ${ }^{9}$ From our current understanding of stellar physics, it is not possible for a $17 M_{\odot}$ single star to shed enough of its hydrogen-rich envelope on the main sequence to avoid the RSG phase (Meynet \& Maeder 2000; the influence of binarity on the evolution of a star of similar mass was explored in the previous section).

If magnetars can be produced from stars which will inevitably suffer core spin-down during their evolution, then perhaps stellar rotation, and in turn initial stellar mass, are not the primary factors in the production of extreme magnetic fields in neutron stars. An alternative theory to the dynamo mechanism is the "fossil-field" scenario, whereby a seed $B$-field is inherited by a newly born star from its natal molecular cloud (e.g., Ferrario \& Wickramasinghe 2005). This explanation is preferred from studies of the energetics of SN remnants associated with magnetars, in which no evidence has been found for the extra energy boost provided by the neutron star's rapid spin-down (such as predicted by the dynamo scenario; Vink \& Kuiper 2006). However, while a handful of massive stars have recently been observed to have magnetic field strengths of $\sim 10^{3} \mathrm{G}$ (Donati et al. 2006; Bouret et al. 2008), it has been noted that flux conservation alone is not sufficient to amplify the $B$-field to that of magnetar levels (Spruit 2008), and no current theory exists for how such fields evolve with the star up to the point of $\mathrm{SN}$.

\footnotetext{
9 However, see also Ott et al. (2006) for calculations on neutron star birth periods.
} 


\section{SUMMARY AND CONCLUSIONS}

We present an imaging and spectroscopic study of the host cluster of the magnetar SGR1900+14, with the purpose of deriving the initial mass of the magnetar's progenitor. From analysis of the two bright RSGs in the cluster, we determine a kinematic distance of $12.5 \pm 1.7 \mathrm{kpc}$ and extinction of $A_{\mathrm{V}}=12.9 \pm 0.5$, which is in good agreement with that previously derived for the magnetar. From the luminosities of the RSGs, we determine an age for the cluster of $14 \pm 1 \mathrm{Myr}$, and a study of the fainter stellar population of the cluster reveals that it is consistent with being coeval to within the errors. Assuming that the SN that created the magnetar occurred very recently in comparison to the age of the cluster, we derive an initial mass of the magnetar's progenitor of $M_{\text {prog }}=17 \pm 2 M_{\odot}$. We find this estimate is insensitive to parameters such as metallicity and the type of evolutionary models used. This result is significantly lower than for other magnetars with progenitor mass estimates, and challenges the hypothesis that very high initial masses $\left(\gtrsim 40 M_{\odot}\right)$ are required to produce magnetars. Instead, we suggest that some other initial parameter, such as magnetic field strength at birth or the presence of a close binary companion, may be the dominant factor in producing a superstrong magnetic field.

We thank Jim Hinton and John Eldridge for useful discussion, and the anonymous referee for helpful comments and suggestions. This work makes use of the UKIDSS survey; the UKIDSS project is defined in Lawrence et al. (2007). UKIDSS uses the UKIRT Wide Field Camera (WFCAM; Casali et al. 2007) and a photometric system described in Hewett et al. (2006). The pipeline processing and science archive are described in Hambly et al. (2008). The material in this work is supported by NASA under award NNG 05-GC37G, through the Long-term Space Astrophysics program. This research was performed in the Rochester Imaging Detector Laboratory with support from a NYSTAR Faculty Development Program grant. Part of the data presented here were obtained at the W. M. Keck Observatory, which is operated as a scientific partnership among the California Institute of Technology, the University of California, and the National Aeronautics and Space Administration. The Observatory was made possible by the generous financial support of the W. M. Keck Foundation. This research has made use of the IDL software package and the GSFC IDL library.

\section{REFERENCES}

Bibby, J. L., Crowther, P. A., Furness, J. P., \& Clark, J. S. 2008, MNRAS, 386, L23

Bouret, J.-C., Donati, J.-F., Martins, F., Escolano, C., Marcolino, W., Lanz, T., \& Howarth, I. D. 2008, MNRAS, 389, 75

Brand, J., \& Blitz, L. 1993, A\&A, 275, 67

Casali, M., et al. 2007, A\&A, 467, 777
Davies, B., Figer, D. F., Kudritzki, R.-P., MacKenty, J., Najarro, F., \& Herrero, A. 2007, ApJ, 671, 781

Davies, B., Figer, D. F., Law, C. J., Kudritzki, R.-P., Najarro, F., Herrero, A., \& MacKenty, J. W. 2008, ApJ, 676, 1016

Donati, J.-F., Howarth, I. D., Bouret, J.-C., Petit, P., Catala, C., \& Landstreet, J. 2006, MNRAS, 365, L6

Duncan, R. C., \& Thompson, C. 1992, ApJ, 392, L9

Eldridge, J. J., Izzard, R. G., \& Tout, C. A. 2008, MNRAS, 384, 1109

Ferrario, L., \& Wickramasinghe, D. T. 2005, MNRAS, 356, 615

Figer, D. F., Najarro, F., Geballe, T. R., Blum, R. D., \& Kudritzki, R. P. 2005, ApJ, 622, L49

Figer, D. F., et al. 2003, ApJ, 599, 1139

Gaensler, B. M., et al. 2005a, Nature, 434, 1104

Gaensler, B. M., McClure-Griffiths, N. M., Oey, M. S., Haverkorn, M., Dickey, J. M., \& Green, A. J. 2005b, ApJ, 620, L95

Gotthelf, E. V., \& Halpern, J. P. 2008, ApJ, 681, 515

Gotthelf, E. V., \& Halpern, J. P. 2009, ApJ, 700, L158

Hambly, N. C., et al. 2008, MNRAS, 384, 637

Hanson, M. M., Conti, P. S., \& Rieke, M. J. 1996, ApJS, 107, 281

Hanson, M. M., Kudritzki, R.-P., Kenworthy, M. A., Puls, J., \& Tokunaga, A. T. 2005, ApJS, 161, 154

Heger, A., Woosley, S. E., \& Spruit, H. C. 2005, ApJ, 626, 350

Helfand, D. J., Gotthelf, E. V., Halpern, J. P., Camilo, F., Semler, D. R., Becker, R. H., \& White, R. L. 2007, ApJ, 665, 1297

Hewett, P. C., Warren, S. J., Leggett, S. K., \& Hodgkin, S. T. 2006, MNRAS, 367,454

Kaplan, D. L., Kulkarni, S. R., Frail, D. A., \& van Kerkwijk, M. H. 2002, ApJ, 566,378

Kouveliotou, C., et al. 1994, Nature, 368, 125

Kouveliotou, C., et al. 1998, Nature, 393, 235

Kouveliotou, C., et al. 1999, ApJ, 510, L115

Kroupa, P. 2001, MNRAS, 322, 231

Lawrence, A., et al. 2007, MNRAS, 379, 1599

Levesque, E. M., Massey, P., Olsen, K. A. G., Plez, B., Josselin, E., Maeder, A., \& Meynet, G. 2005, ApJ, 628, 973

Lucas, P. W., et al. 2008, MNRAS, 391, 136

Mereghetti, S. 2008, A\&AR, 15, 225

Messineo, M., Figer, D. F., Davies, B., Rich, R. M., Valenti, E., \& Kudritzki, R. P. 2008, ApJ, 683, L155

Meynet, G., \& Maeder, A. 2000, A\&A, 361, 101

Meynet, G., Maeder, A., Schaller, G., Schaerer, D., \& Charbonnel, C. 1994, A\&AS, 103, 97

Morris, T., \& Podsiadlowski, P. 2006, MNRAS, 365, 2

Muno, M. P., et al. 2006, ApJ, 636, L41

Ott, C. D., Burrows, A., Thompson, T. A., Livne, E., \& Walder, R. 2006, ApJS, 164,130

Podsiadlowski, P., Joss, P. C., \& Hsu, J. J. L. 1992, ApJ, 391, 246

Rieke, G. H., \& Lebofsky, M. J. 1985, ApJ, 288, 618

Schaerer, D., Charbonnel, C., Meynet, G., Maeder, A., \& Schaller, G. 1993 A\&AS, 102, 339

Schaller, G., Schaerer, D., Meynet, G., \& Maeder, A. 1992, A\&AS, 96, 269

Spruit, H. C. 2008, in AIP Conf. Ser. 983, 40 Years of Pulsars: Millisecond Pulsars, Magnetars and More, ed. C. Bassa et al. (Melville, NY: AIP), 391 Testa, V., et al. 2008, A\&A, 482, 607

Thompson, T. A., Chang, P., \& Quataert, E. 2004, ApJ, 611, 380

Thompson, C., \& Duncan, R. C. 1995, MNRAS, 275, 255

Vink, J., \& Kuiper, L. 2006, MNRAS, 370, L14

Vrba, F. J., Henden, A. A., Luginbuhl, C. B., Guetter, H. H., Hartmann, D. H., \& Klose, S. 2000, ApJ, 533, L17

Vrba, F. J., et al. 1996, ApJ, 468, 225

Wachter, S., Ramirez-Ruiz, E., Dwarkadas, V. V., Kouveliotou, C., Granot, J., Patel, S. K., \& Figer, D. 2008, Nature, 453, 626

Wallace, L., \& Hinkle, K. 1996, ApJS, 103, 235 